\title{
Colectivo de Profesores-con-Doblado-de-Papel en Tareas de Geometría Escolar
}

\section{Teachers-with-Paper-Folding Collective in School Geometric Tasks}

\author{
Zaida Santa-Ramírez \\ ORCID iD 0000-0003-0272-2405 \\ Carlos Jaramillo-López ${ }^{* *}$ \\ ORCID iD 0000-0002-3937-5032 \\ Élgar Gualdrón-Pinto ${ }^{* * *}$ \\ ORCID iD 0000-0002-4081-2092
}

\begin{abstract}
Resumen
La revisión de la literatura permite inferir que, en el momento, no se ha abordado un estudio que dé cuenta de la caracterización de la producción de conocimiento geométrico escolar, que parece propiciarse al interior de un colectivo particular de profesores-con-doblado-de-papel y que puede evidenciarse mediante el desarrollo de talleres, donde se implementan tareas de formación de profesores basadas en construcciones con doblado de papel. Por lo tanto, este artículo pretende mostrar, a través de un estudio cualitativo, que la producción de conocimiento geométrico escolar parece emerger de un colectivo de profesores-con-doblado-de-papel; en esta perspectiva, la investigación se fundamenta en el constructo teórico seres-humanos-con-medios. El diseño metodológico presenta una tarea de formación relacionada con la división de un segmento en media y extrema razón con doblado de papel, junto con su respectivo análisis previo, del cual surgen algunas categorías emergentes preliminares que pueden caracterizar dicha producción de conocimiento; es decir, esta última se asocia con los siguientes aspectos: uso del doblado de papel como medio para visualizar, experimentar y comprender conceptos; interacciones del colectivo de profesores-con-doblado-de-papel y, finalmente, con las reflexiones en torno a la práctica pedagógica.
\end{abstract}

Palabras clave: Colectivo de profesores-con-doblado-de-papel. Doblado de papel. Producción de conocimiento geométrico escolar. Seres-humanos-con-medios.

\begin{abstract}
The literature review allows us to infer that there is not a study in which one has approached the characterization of the school geometric knowledge production, which seems to be generated within a particular teachers-withpaper-folding collective and it can be evidenced by the development of workshops, where teacher training tasks are implemented based on paper folding constructions. Therefore, the qualitative study described in this article

\footnotetext{
* Doctora en Educación de la Universidad de Antioquia (UdeA). Profesora ocasional de tiempo completo del Tecnológico de Antioquia IU (TdeA), Medellín, Antioquia, Colombia. Dirección postal: calle 78B \# 72 ${ }^{\mathrm{a}}-220$, Medellín, Antioquia, Colombia, C.P: 050034. E-mail: zaida.santa@tdea.edu.

${ }^{* *}$ Doctor en Ciencias Matemáticas de la Universidad Politécnica de Valencia (UPV), Valencia, España. Profesor asociado de la Universidad de Antioquia (UdeA), Medellín, Antioquia, Colombia. Dirección postal: calle 70 \# 52 -21, Medellín, Antioquia, Colombia, C.P: 050010. E-mail: carlos.jaramillo1@ udea.edu.co.

*** Doctor en Didáctica de las Matemáticas de la Universidad de Valencia (UV), Valencia, España. Profesor asociado de la Universidad de Pamplona, Pamplona, Norte de Santander, Colombia. Dirección postal: calle 5 \# 4 - 19, Pamplona, Norte de Santander, Colombia, C.P: 543050. E-mail: egualdron@ unipamplona.edu.co.
} 
shows through specific cases, that school geometrical knowledge production seems to emerge from a teacherswith-paper-folding collective; therefore, the research is based on the Humans-with-media construction. The design presents a training teachers' task, associated with the division of a segment in extreme and mean ratios, with paper folding, along with their respective prior methodological analysis, from which emerge some preliminary categories that can characterize this said knowledge production; these categories are: use of paper folding as a media to visualize, experience and understand concepts; interaction of teachers-with-paper-folding collective and, finally, the reflections about pedagogical practice.

Keywords: Teachers-with-paper-folding collective. Paper Folding. School geometric knowledge production. Humans-with-media.

\section{Introducción}

En la perspectiva de Villarreal (2012), la cognición humana se fundamenta en la construcción social del conocimiento mediante el uso de medios o artefactos. Esta afirmación parece esclarecerse, particularmente, en nuestra práctica pedagógica, cuando presenciamos que durante la puesta en escena de talleres, mediante la implementación de construcciones con doblado de papel, los profesores construyen, consolidan o transforman sus conocimientos sobre geometría escolar.

Moreira y David (2005, p. 20, traducción libre) afirman que "la matemática escolar incluye tanto saberes producidos y movilizados por los profesores de matemática en su acción pedagógica en el aula de clase, como los resultados de investigaciones que se refieren al aprendizaje y a la enseñanza escolar de conceptos matemáticos, técnicas, procesos etc.”. Del mismo modo, en la línea de estos autores, se entenderá que la geometría escolar es un subconjunto de la matemática escolar y, por lo tanto, incluirá no solo saberes geométricos generados o consolidados por los profesores de geometría en su práctica pedagógica, sino también los resultados de investigaciones relacionados con los procesos de enseñanza y aprendizaje de conceptos y procedimientos geométricos.

Considerando los aportes de Villarreal (2012), y en la perspectiva de Borba y Villarreal (2005), quienes también hacen hincapié en que la producción de conocimiento se construye en colectivos de seres-humanos-con-medios, es importante resaltar que algunos miembros de grupos de investigación brasileiros, como los del Grupo de Pesquisa em Informática, outras Mídias e Educação Matemática - GPIMEM, de Rio Claro, São Paulo, han intentado hacer una descripción aproximada sobre la producción de conocimiento matemático que emerge de colectivos de profesores o estudiantes, cuando usan determinados medios tecnológicos, como softwares educativos, celulares, calculadoras, tablets, entre otros.

Pero se puede inferir, de acuerdo a la revisión de la literatura existente al respecto, que aún no se ha abordado un estudio que dé cuenta de la caracterización de la producción de 
conocimiento geométrico escolar, que parece propiciarse al interior de un colectivo de profesores-con-doblado-de-papel y que puede evidenciarse mediante el desarrollo de talleres, donde se implementan tareas ${ }^{1}$ de formación de profesores basadas en construcciones con doblado de papel.

Dado que los medios utilizados condicionan la naturaleza del conocimiento producido (BORBA; VILLARREAL, 2005), la caracterización de los procesos de producción de conocimiento geométrico escolar, que surgen de colectivos-con-doblado-de-papel, sería diferente a la de los colectivos-con-regla-y-compás o colectivos-con-software-educativos. De hecho, esa caracterización también estaría condicionada por el colectivo de personas mismo. Es decir, "una nueva tecnología resulta en un nuevo colectivo que produce nuevos conocimientos, que son cualitativamente diferentes a los conocimientos producidos por otros colectivos" (BORBA; VILLARREAL, 2005, p. 24).

Por lo tanto, el estudio Producción de conocimiento geométrico escolar en un colectivo de profesores-con-doblado-de-papel, que se desarrolló en el marco del programa de Doctorado en Educación, de la Facultad de Educación de la Universidad de Antioquia, pretendió analizar y caracterizar la producción de conocimiento geométrico escolar que surgió de un colectivo de profesores-con-doblado-de-papel. Es decir, dio respuesta a la pregunta: ¿cómo producir conocimiento geométrico escolar en un colectivo de profesores-con-doblado-de-papel?

En los siguientes apartados, presentamos algunos aspectos de la fundamentación teórica y metodológica del estudio, considerando las ideas fundantes del constructo teórico sereshumanos-con-medios de Borba y Villarreal (2005) y la descripción del paradigma cualitativo de investigación, en el cual se basa el diseño investigativo.

Posteriormente, explicaremos la tarea de formación media y extrema razón, que se desarrolló en un colectivo particular de profesores-con-doblado-de-papel. En el cuarto apartado, mostramos algunos resultados, producto del trabajo de campo con los profesores del colectivo y, finalmente, presentamos unas primeras categorías que surgen de un análisis preliminar de la información y que nos permitirán inferir conclusiones parciales del estudio, relacionadas con la pregunta de investigación.

\section{Referente teórico: seres-humanos-con-medios}

\footnotetext{
${ }^{1}$ Las tareas de formación involucran, en la perspectiva de Ponte et al. (2009), problemas y actividades geométricas, que son ofrecidas a los profesores para que tengan la oportunidad de profundizar en los conocimientos que deben enseñar a sus estudiantes y en la manera como pueden enseñarlos.
} 
Marcelo Borba y Mónica Villarreal presentaron, en el año 2005, el libro oficial que consolida un constructo teórico llamado seres-humanos-con-medios, en el cual analizan de qué manera el conocimiento matemático es producto de las interacciones entre un grupo de humanos pensantes con determinados medios. En este sentido, se habla de "un colectivo pensante de humanos-con-medios" (VILLA-OCHOA; RUIZ, 2010, p. 517).

Este estudio está basado en el constructo teórico mencionado, porque concordamos que la producción de conocimiento es de naturaleza social, en tanto que se genera en colectivos de seres humanos cuando utilizan determinados medios o artefactos. De acuerdo con Borba, Scucuglia y Gadanidis (2014), el conocimiento es producido por colectivos de seres-humanoscon-medios, teniendo en cuenta que los medios pueden ser desde un lápiz y un papel, hasta un software, internet, entre otros. Es decir, el conocimiento es generado y moldeado por humanos y tecnologías, situados en los respectivos contextos históricos; esos colectivos producen nuevas tecnologías y nuevos conocimientos, los cuales caracterizan el significado de ser humano en un momento histórico determinado (BORBA, 2012).

La expresión seres-humanos-con-medios fue creada como una metáfora para hacer alusión a la relación entre quien conoce y los medios (BORBA, 1999), considerando como base teórica fundamental las nociones de tecnologías de la inteligencia de Tikhomirov (1981) y colectivos pensantes de Lévy (1993). También, en torno al constructo teórico seres-humanoscon-medios, es clara la influencia de la teoría de la actividad y de diversas tendencias de investigación como la experimentación, la visualización, el uso de calculadoras gráficas y software, el uso de internet en Educación Matemática, modelación matemática, formación de profesores, metodología de investigación cualitativa, dimensiones políticas y educación matemática crítica, entre otros (BORBA; SCUCUGLIA; GADANIDIS, 2014). Es importante aclarar que algunos de estos aspectos no serán abordados en este artículo.

La metáfora que establece la relación entre el que conoce y los medios puede ser ampliada a los sistemas ser-humano-oralidad, ser-humano-escritura o ser-humano-informática, en los cuales el pensamiento es colectivo, de acuerdo con Lévy (1993) y Borba (1999). Por lo tanto, los medios no son solo molduras, sino que son parte activa del pensamiento. Esto es, el pensamiento es condicionado, no determinado, por las diferentes técnicas desarrolladas a lo largo de la historia (BORBA, 1999).

Por lo tanto, Borba (1999) y Souto (2013) utilizan el término medios para referirse tanto a las tecnologías materiales (herramientas, instrumentos, cosas, entre otras), como a las inmateriales (oralidad, escritura, informática, pensamiento, entre otras). Por su parte, Villarreal y Borba (2010) afirman que el medio es cualquier clase de herramienta, dispositivo, equipo, 
instrumento, artefacto o material como resultado del desarrollo tecnológico y que dentro del sistema seres-humanos-con-medios, permite la reorganización del pensamiento.

De acuerdo con lo anterior, para el constructo teórico seres-humanos-con-medios, la producción de conocimiento se debe entender como pensar con medios; es decir, los medios (tecnologías materiales o inmateriales) deben ser considerados como parte constitutiva de esa producción, en la medida en que a estos se les atribuye un papel más relevante en la producción de conocimiento y dejan de ser un simple soporte del mensaje a través de (BORBA; VILLARREAL, 2005; SOUTO, 2013).

En consecuencia, el medio no se puede entender como cualquier dispositivo que esté al alcance humano; el medio se debe entender de manera más general, más global, como aquel artefacto o recurso (tecnología material o inmaterial) que permite la reorganización del pensamiento. Por lo tanto, en este estudio, tanto el doblado de papel, como el lenguaje verbal o escrito y los procesos de experimentación y visualización, son tomados como medios, los cuales son parte activa del pensamiento de los profesores del colectivo.

\section{Metodología}

\subsection{Paradigma y tipo de estudio}

Considerando que el estudio mencionado pretende analizar cómo se produce conocimiento geométrico escolar en un colectivo de profesores-con-doblado-de-papel y, asumiendo que esta producción es una construcción de tipo social (VILLARREAL, 2012), la investigación se guía bajo un paradigma cualitativo. En palabras de Borba (2012), el conocimiento y, en particular, la producción de este, es un esfuerzo humano atravesado por la subjetividad, que debe estar representado por una investigación cualitativa. Por lo tanto, se interpreta y analiza un fenómeno de tipo social (interacciones entre las personas y los medios) que "no sigue un proceso claramente definido" (HERNÁNDEZ; FERNÁNDEZ; BAPTISTA, 2006, p. 8).

Durante el proceso de recolección de información, se ha hecho hincapié en que el investigador cualitativo debe seguir tomando decisiones, "modificando, cambiando, alterando o rediseñando su trabajo" (RODRÍGUEZ; GIL; GARCÍA, 1999, p. 74), incluso en el momento del trabajo de campo, lo que hace que el estudio sea flexible para poder responder a la pregunta de investigación y dar consecución a los objetivos planteados. 
Desde esta perspectiva, la información se ha recolectado a través de los siguientes instrumentos: (i) observaciones, las cuales ofrecen una descripción relativamente incuestionable de la realidad; (ii) material de los profesores que ha emergido de todas las actividades propuestas; (iii) diarios de campo o bitácoras, tanto de los investigadores, como de los participantes, que contienen sus producciones conceptuales, retrocesos, conjeturas visuales, experiencias vividas, transformaciones posibles en el aula de clase, propuestas de enseñanza, entre otras, que se han elaborado dentro del colectivo a través de las actividades desarrolladas.

De acuerdo con lo anterior, se abordó el siguiente proceso investigativo: diseño y revisión de tareas de formación con doblado de papel, surgidas del diálogo continuo con los colectivos; puesta en marcha de dichas tareas al interior de los colectivos, considerando tanto aspectos disciplinares de la geometría, como el análisis de su enseñanza (geometría escolar); análisis de la producción de conocimiento geométrico escolar que emerge de colectivos de profesores-con-doblado-de-papel; evaluación y refinación de las tareas de formación, considerando las experiencias vividas y el diálogo constante de colectivos de profesores (SANTA; JARAMILLO, 2015).

Por lo tanto, el diseño metodológico que orienta este estudio es una investigación de diseño o investigación basada en diseño (design research), desde las ideas de Molina et al. (2011). Estos autores precisan que la investigación de diseño o investigación basada en diseño, es un paradigma de investigación principalmente cualitativo, cuyo objetivo es analizar el aprendizaje en contexto a través de: (1) diseño y estudio de formas muy particulares de aprendizaje; (2) estrategias y herramientas usadas para la enseñanza; (3) el análisis de la naturaleza sistémica del aprendizaje, la enseñanza y la evaluación (MOLINA et al., 2011).

La producción de conocimiento geométrico escolar en colectivos de profesores-condoblado-de-papel, que puede surgir de la puesta en marcha de tareas de formación planteadas, se puede convertir en un escenario de aprendizaje matemático que, de acuerdo con Borba, Scucuglia y Gadanidis (2014), se asocia con la producción de conocimientos matemáticos, con el pensamiento matemático y con la producción de significados. Este escenario puede ser un "ambiente de aprendizaje que sirve como contexto para la investigación" (MOLINA et al., 2011, p. 76).

El análisis continuo que se realizó durante el trabajo de campo y el análisis retrospectivo, pueden mejorar y refinar el producto que, en este caso, era el conjunto de tareas generadas, para explicar su funcionalidad y sugerir formas de adaptación a otros contextos o colectivos. Sin embargo, más allá del diseño y evaluación de estas actividades, la intención es explicar cómo se produce conocimiento geométrico escolar en colectivos de profesores-con-doblado-de-papel, 
a través de las interacciones que se posibilitan durante el desarrollo de las tareas, lo que permite incluir y reflejar un entendimiento de las relaciones entre el constructo teórico seres-humanoscon-medios, la práctica que se lleva a cabo en el trabajo de campo y los instrumentos diseñados (tareas).

\subsection{Camino metodológico: media y extrema razón como tarea de formación}

Una de las tareas de formación diseñadas como propuesta para los profesores, con el propósito de dar consecución al objetivo general, es la relacionada con la construcción de la media y extrema razón de un segmento, a través de la geometría del doblado de papel (SANTA; JARAMILLO, 2010). Con esta tarea se busca propiciar procesos de interacción dentro del colectivo, que permitan, a su vez, producción de conocimiento geométrico escolar; esta se dividió en cuatro momentos (SANTA; JARAMILLO; BORBA, 2015):

Momento 1. Construcción. A continuación, se presentan los pasos para la construcción de la media y extrema razón de un segmento, con sus respectivas representaciones visuales en doblado de papel (Figuras 1 - 8). Considerando los procedimientos brindados por Row (1966), nos permitimos redactar instrucciones geométricas y hacer interpretaciones que nos lleven a generar construcciones en el marco de los objetivos del estudio. Es importante mencionar que los dobleces se resaltan para facilitar la visualización y comprensión de los pasos de la construcción.

Paso 1 (Figura 1): tome una hoja de papel de forma cuadrada y nómbrela ABCD. (SANTA; JARAMILLO; BORBA, 2015).

Paso 2 (Figura 2): busque el punto medio del segmento $\overline{A D}$ llevando $\overline{A B}$ sobre $\overline{D C}$ (media paralela), pero no haga todo el doblez sino solo una señal. Nómbrelo M. (SANTA; JARAMILLO; BORBA, 2015).

Paso 3 (Figura 3): realice el doblez $\overline{M B}$ y desdoble. (SANTA; JARAMILLO; BORBA, 2015).

Paso 4: encuentre la bisectriz del ángulo < AMB, es decir, lleve $\overline{A M}$ sobre $\overline{M B}$ y haga el doblez (Figura 4). Marque el punto A sobre $\overline{M B}$ y nómbrelo A'. Desdoble (Figura 5). (SANTA; JARAMILLO; BORBA, 2015).

Paso 5 (Figura 6) encuentre la bisectriz del ángulo < ABM. Esto es, lleve $\overline{A B}$ sobre

$\overline{M B}$. Realice el doblez respectivo. (SANTA; JARAMILLO; BORBA, 2015). 
Paso 6: marque el punto $\mathrm{A}^{\prime}$ sobre $\overline{A B}$ y nómbrelo $\mathrm{X}$ (Figura 7). Así se garantiza que $\overline{B X} \cong \overline{B A^{\prime}}$ (Figura 8). (SANTA; JARAMILLO; BORBA, 2015).

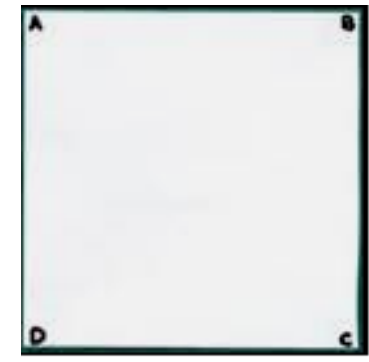

Figura 1 - Paso 1

Fuente: Santa, Jaramillo y Borba (2015).

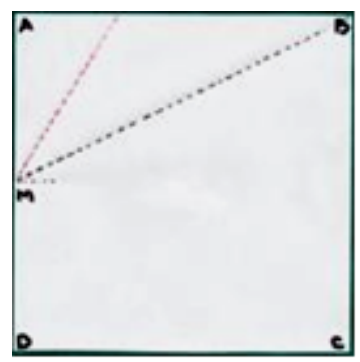

Figura 4 - Paso 4a Fuente: Santa, Jaramillo y Borba (2015).

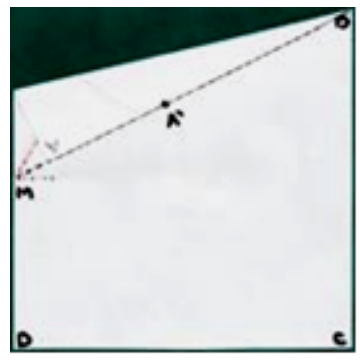

Figura 7 - Paso 6a Fuente: Santa, Jaramillo y Borba (2015)

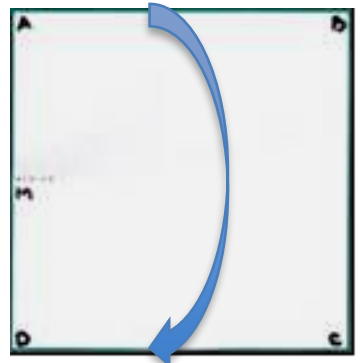

Figura 2 - Paso 2

Fuente: Santa, Jaramillo y Borba (2015).

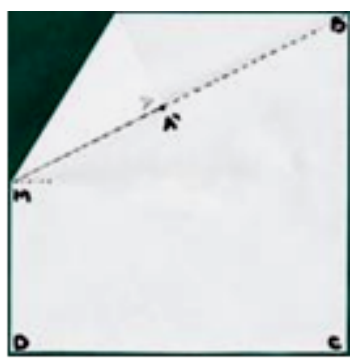

Figura 5 - Paso $4 \mathrm{~b}$

Fuente: Santa, Jaramillo y Borba (2015).

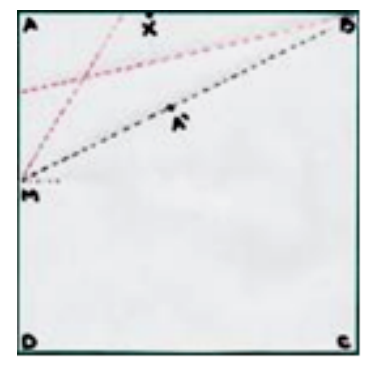

Figura 8 - Paso 6b

Fuente: Santa, Jaramillo y Borba (2015).

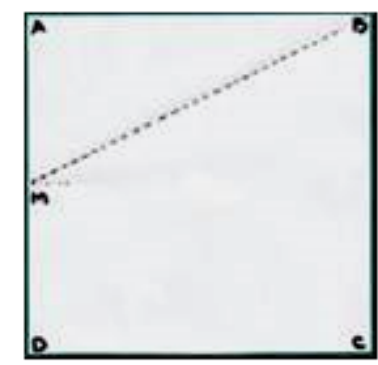

Figura 3 - Paso 3 Fuente: Santa, Jaramillo y Borba (2015).

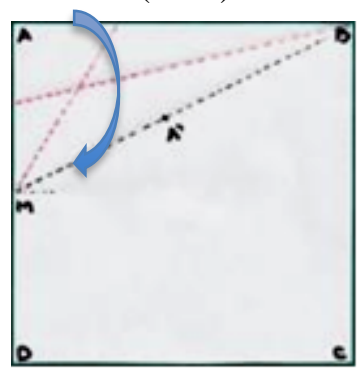

Figura 6 - Paso 5

Fuente: Santa, Jaramillo y Borba (2015).

Momento 2. Análisis geométrico de la media y extrema razón. Posterior a la construcción de la media y extrema razón (Figura 9), se les solicitó al colectivo de profesores analizar el proceso abordado para identificar diversas implicaciones geométricas. Adicionalmente, se les propuso que expliquen, mediante argumentos de tipo matemático, que el punto $\mathrm{X}$ divide el segmento $\overline{A B}$ en media y extrema razón, a partir de la visualización que

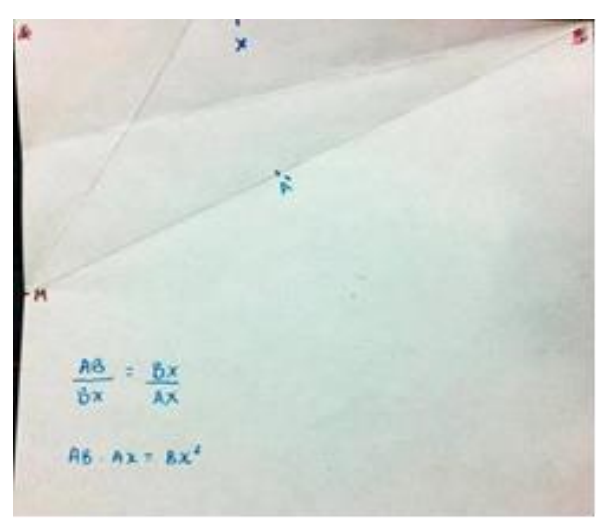

Figura 9 - Material de la profesora Natalia Fuente: construcción personal. 
permite la construcción con doblado de papel. Es decir, debían verificar que la proporción: $\frac{\overline{A B}}{\overline{X B}}=\frac{\overline{X B}}{\overline{A X}} \leftrightarrow \overline{A B} \times \overline{A X}=\overline{X B}^{2}$

Momento 3. Relación de construcciones. Los investigadores, usuarios del software Geogebra, propusieron observar el proceso de construcción seguido mediante el doblado de papel, para establecer cuáles podrían ser los pasos correspondientes que deberían abordarse a través de este software, de tal manera que se pudiera lograr una construcción euclidiana similar. El propósito era que los profesores pudieran determinar que la construcción euclidiana, realizada en Geogebra, es equivalente, de manera implícita, a la realizada con doblado de papel (Figuras 9 y 10).

Momento 4. Análisis didáctico. Finalmente, los profesores analizaron los procesos de construcción y su pertinencia en el aula de clase para, posteriormente, pensar en la posibilidad de generar actividades que permitan a sus estudiantes la producción de conocimiento geométrico mediante el doblado de papel.

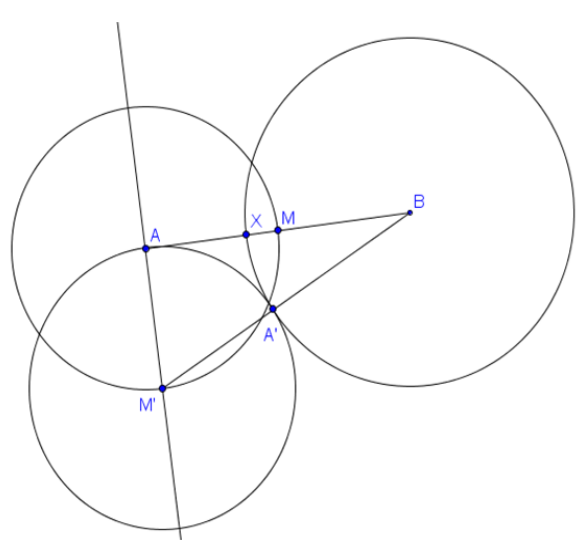

Figura 10 - Construcción euclidiana Fuente: construcción de los autores en Geogebra.

\section{Resultados y su análisis}

El análisis de lo ocurrido durante el desarrollo de la tarea de formación de media y extrema razón de un segmento, por el colectivo de profesores-con-doblado-de-papel, se presenta en dos secciones. La primera muestra algunas evidencias que surgieron de la transcripción de los videos, de las bitácoras de los profesores y de sus construcciones con doblado de papel. La segunda, permite observar tres posibles categorías emergentes derivadas del análisis de las evidencias, que podrían responder, en parte, la pregunta de investigación del estudio.

\subsection{Algunas evidencias}


La tarea de formación relacionada con la media y extrema razón de un segmento, fue realizada por las profesoras: Natalia, Elizabeth y Vicky², además de los investigadores. Es importante mencionar que el colectivo estaba conformado, inicialmente, por siete profesores; sin embargo, al transcurrir el tiempo y al incrementarse sus responsabilidades en la Institución Educativa a la que pertenecen, el colectivo se fue reduciendo y, al final, contó con tres participantes mujeres.

Tanto la profesora Natalia, como la profesora Elizabeth, son del área de matemáticas; mientras que la profesora Vicky es del área de química. A las tres se les notó entusiasmo y motivación para participar de las diez sesiones programadas, las cuales se llevaron a cabo en la Institución Educativa donde laboran. En este sentido, los investigadores estuvimos inmersos en el contexto social y cultural de las profesoras; de hecho, nos sentimos reconocidos como colegas porque cuando trabajamos de manera conjunta con el colectivo, se evidenció que las interacciones no se basaban en relaciones de autoridad o jerarquía.

El encuentro, cuyo tema principal fue la división de un segmento en media y extrema razón, inició con un conversatorio sobre el significado de este procedimiento. La profesora Natalia mencionó que se relacionaba con proporciones. Las otras dos profesoras no sabían aún de qué se estaba hablando. Por lo tanto, se reformuló la pregunta, haciendo referencia a la sección áurea o divina proporción. De esta manera, la profesora Elizabeth afirmó que había realizado actividades con los estudiantes, en el aula de clase, relacionadas con algunas medidas del cuerpo humano, pero con el único propósito de determinar el número de oro (o número Phi). La profesora Vicky mencionó que se relacionaba con el ciclo lunar; mientras que la profesora Natalia explicitó que se relacionaba con algunas sucesiones, como la de Fibonacci. Posteriormente, establecimos que si el punto $\mathrm{X}$ divide el segmento $\overline{A B}$ en media y extrema razón, entonces se cumple la proporción mencionada en el momento dos.

Al finalizar el conversatorio, realizamos la construcción de la media y extrema razón, indicando los pasos a seguir en una hoja de papel y mediante una presentación con diapositivas, que contenía las respectivas imágenes visuales de cada paso (Figuras 1 - 8). Cuando se hacía algún doblez, se preguntaba por las implicaciones geométricas del mismo (mediatriz, bisectriz, punto medio, traslado de medidas y de segmentos, entre otras). Además, si una instrucción no era entendida, alguna de las profesoras del colectivo asumía la vocería y explicaba lo que estaba

\footnotetext{
2 Algunas de las profesoras, por voluntad propia y considerando los aspectos éticos sobre confidencialidad, decidieron usar como seudónimo su propio nombre. Otras, eligieron el seudónimo que más les llamaba la atención.
} 
ocurriendo. Posterior a la construcción (Figura 9), se les preguntó a las profesoras cómo se podía verificar la proporción en cuestión. Para ello, se presentó el siguiente diálogo:

1. Investigadora Z: [...] Debemos comprobar que esa relación es verdad. Pero entonces ¿qué sabemos?

2. Hay que llegar a esto, pero entonces vamos a sacar cosas. Vamos a sacar ciertas hipótesis ahí.

3. ¿Qué sabemos de la construcción? ¿Qué relaciones podemos sacar ahí?

4. Elizabeth: $Q u e \overline{B X}$ es más grande que $\overline{A X}$. Que $\overline{A B}$ es más grande.

5. Investigadora $\mathrm{Z}$ : $O$ sea, $\overline{A B}$ es más grande... ¿Qué tal si le damos una medida a $\overline{A B}$ ?

6. Elizabeth: $L$

7. Investigadora Z: L, se llama L. Mide L.

8. Elizabeth: $\overline{A B}$ es $L$.

9. Vicky: $\overline{A M}$ es L a la 2. Ehh, L/2.

10.Investigadora Z: Muy bien. ¿Qué más? ¿Qué hicimos? Trasladamos quién sobre quién... 11. Natalia: $\overline{A M}$ sobre $\overline{M B}$.

12.Investigadora $\mathrm{Z}:$ [...] Si este es $\overline{A M}$ que mide L/2, entonces $\overline{M A^{\prime}}$ ¿qué es? 13. Elizabeth: $L / 2$

14.Investigadora Z: entonces $\overline{M A^{\prime}}$ también mide L/2. Muy bien. [...] ¿Qué más?

15. No le demos valores a $\overline{A^{\prime} B}$ y a $\overline{B X}$, pero ¿qué sabemos de la relación entre $\overline{B A^{\prime}}$...?

16. Vicky: $\overline{B X}$ es igual a $\overline{A^{\prime} B}$.

17.Investigadora $\mathrm{Z}: \mathrm{Ok}, \overline{B X}$ es congruente con $\overline{A^{\prime} B}$. [...] ¿Cuánto medirá $\overline{M B}$ ?

18. Elizabeth: Mide $L / 2$ más $\overline{A^{\prime} B}$. Le restamos a $\overline{M B}$, L/2 y ahí sabemos $\overline{A^{\prime} B}$

19.Investigadora Z: Muy bien. [...] Si yo busco qué es $\overline{A^{\prime} B}$, encuentro...

20. Natalia: $\overline{B X}$.

21. Investigadora $\mathrm{Z}$ : ¿Cómo relaciono $\overline{M B}$ en términos de L? ¿Solo L? Pero, ¿qué figura es esa?

22.Elizabeth: un triángulo rectángulo.

23.Investigadora Z: [...] De ese triángulo rectángulo, ¿cuál es la medida de sus catetos?

24. Elizabeth: $\overline{A B}$ y $\overline{A M}$ y encuentro la hipotenusa $\overline{M B} \cdot \overline{A B}$ mide $L$ y $\overline{A M}$ mide $L / 2$.

25.Investigadora $\mathrm{Z}:[. .$.$] O sea que puedo buscar \overline{M B}$...

Después de establecer algunas hipótesis, el colectivo de profesoras se dispuso a encontrar las medidas de algunos segmentos, para poder verificar la relación que surge de la división de un segmento en media y extrema razón. Posterior a la verificación del algoritmo de la divina proporción, se utilizó el software Geogebra (Figura 10) para recrear de manera conjunta con el colectivo la construcción que se hizo con doblado de papel. Para ello, se construyó un segmento $\overline{A B}$; luego, se buscó el punto medio $\mathrm{M}$ de $\overline{A B}$ y por A, se trazó una perpendicular al segmento; se trasladó la medida del segmento $\overline{M A}$ a la perpendicular tomando como referencia el punto A y utilizando la opción circunferencia (ese punto se llamó M'); se trazó el segmento $\overline{M^{\prime} B}$. Nuevamente, teniendo como referencia el punto M', se usó la herramienta circunferencia y se trasladó la medida $\overline{M^{\prime} A}$. El punto de corte entre este arco y el 
segmento $\overline{M^{\prime} B}$ se llamó $\mathrm{A}^{\prime}$. Finalmente, con centro en $\mathrm{B}$, se trasladó la medida $\overline{B A^{\prime}}$ al segmento $\overline{A B}$, ubicándose el punto $\mathrm{X}$.

De acuerdo con las discusiones del colectivo de profesoras, se determinó que la construcción que se hacía mediante el doblado de papel es la misma construcción euclidiana. $\mathrm{Al}$ respecto, la profesora Elizabeth manifestó:

[...] yo diría que el análisis comprensivo del doblado de papel me lleva a poder graficar utilizando el asistente [...] y se hace doble aprendizaje porque entonces yo estoy mirando aquí [señala la hoja] y estoy mirando allá [mira la pantalla] y busco otras opciones, otras estrategias para poder llegar a... sin hacerlo simplemente haga allí, haga allá, sin ninguna razón. (Profesora Elizabeth, Transcripción del cuarto episodio, 2016).

Para finalizar la tarea de formación, se les preguntó a las profesoras: ¿qué les pareció esa comparación entre el doblado de papel y la geometría euclidiana? ¿Qué opinan ustedes al respecto? ¿Qué podemos hacer nosotros en el aula de clase? La profesora Natalia tomó la palabra y explicó:

[...] a pesar de que uno lo trabaje aquí [señala la hoja] y sea evidente, comprenda y demuestre, esto [señalando la pantalla] es como un paso más a la formalización [...] para que vean que esto que se ha hecho aquí [señala la hoja] es formal [...] Creo que es una validación necesaria. (Profesora Natalia, Transcripción del cuarto episodio, 2016).

Para completar la respuesta anterior, la profesora Elizabeth mencionó:

$Y$ se fortalece el concepto. De todas maneras, lo que se ve acá y se dice, dentro del esquema ya gráfico, en el plano, también le ayuda a fortalecer y a... a fortalecer otros conceptos desde el uso de las herramientas que se tienen. Porque, de igual manera, por ejemplo, el uso del compás $y$ del transportador no es muy amigable para los muchachos, entonces eso también ayuda para que ellos crean la validez que tienen esos instrumentos a la hora de comprobar ciertas cosas en geometría.

(Profesora Elizabeth, Transcripción del cuarto episodio, 2016).

\subsection{Análisis: primeras categorías emergentes}

Durante el desarrollo del trabajo de campo se consideraron unidades de análisis aquellos episodios en los que es posible evidenciar producción de conocimiento geométrico escolar, como resultado de las interacciones del colectivo de profesores-con-doblado-de-papel. El análisis de estos sucesos nos permitió percibir aspectos que pueden aclarar la producción de conocimiento geométrico escolar, algunos de los cuales están relacionados con el doblado de papel, el colectivo de profesores-con-doblado-de-papel y la reflexión en torno a la profesión; estos tres aspectos se describen a continuación como indicios de categorías que han emergido de las interacciones de ese colectivo particular de profesores con el medio doblado de papel, en 
lo referente a la tarea de formación para la construcción de la media y extrema razón de un segmento.

Indicio de categoría 1: uso del doblado de papel como medio para visualizar (generar conjeturas), experimentar y comprender conceptos.

El colectivo de profesoras ha encontrado en el doblado de papel un medio que permite construir figuras, visualizar sus mosaicos de pliegues para generar conjeturas visuales, permite experimentar y crear nuevos dobleces, para hacer mostraciones ${ }^{3}$ y probar dichas conjeturas. Frente a esta situación, la profesora Natalia afirmó que (con relación a la Figura 12):

$[\ldots]$ el trabajo de conceptos o construcciones tan abstractas [...] están al alcance de trabajarse en el aula con el doblado de papel como una herramienta muy segura, de acceso a todos y de fácil comprensión.

(Profesora Natalia, 2016).

En este sentido, Santa y Jaramillo (2010) afirman que el doblado de papel es una alternativa para mejorar los procesos de razonamiento en geometría, porque permite procesos de visualización y de experimentación, que posibilitan manipular una hoja de papel para generar dobleces, lanzar conjeturas y justificar, de manera formal, las construcciones realizadas.

Frente a la visualización, Borba, Scucuglia y Gadanidis (2014) mencionan que esta se torna en un proceso fundamental de pensamiento matemático, dado que envuelve un esquema mental que representa la información visual o espacial; así mismo, es un proceso de formación de imágenes que posibilita la entrada en escena de representaciones de objetos matemáticos para pensar matemáticamente, y ofrece medios para que las conexiones entre las representaciones puedan ocurrir.

En particular, se pudo evidenciar que las profesoras lograron establecer relaciones entre algunas implicaciones geométricas euclidianas con los pasos concretos de la construcción; por ejemplo, cuando la profesora Elizabeth mencionó que se forma un triángulo rectángulo en la construcción de la media y extrema razón (línea 22 del diálogo) o cuando se encontró el algoritmo para mostrar la divina proporción. Se pudo evidenciar, a su vez, que las profesoras manifestaron conjeturas visuales, como por ejemplo,

Le restamos a $\overline{M B}, L / 2$ y ahí sabemos $\overline{A^{\prime} B}$ (Profesora Elizabeth, línea 18 del diálogo, 2016), o cuando la profesora Elizabeth mencionó que la medida de los catetos del triángulo rectángulo son " $\overline{A B}$ y $\overline{A M}$ y encuentro la hipotenusa $\overline{M B}$. $\overline{A B}$ mide L y $\overline{A M}$ mide L/2" (Profesora Elizabeth, línea 24 del diálogo, 2016).

\footnotetext{
${ }^{3}$ Mostración: “acercamiento de carácter operatorio y multisensorialmente tangible a conceptos científicos de tipo matemático, físico, biológico o químico” (MONSALVE; JARAMILLO, 2003, p. 16).
} 
Como se precisó anteriormente, un aspecto importante del doblado de papel es el proceso de experimentación, que se puso de manifiesto en el diálogo que se suscitó para establecer las relaciones geométricas en los pasos seguidos y poder encontrar la proporción. Estas relaciones fueron: “ $\overline{A M}$ sobre $\overline{M B}$ ” (línea 11 del diálogo), idea expuesta por la profesora Natalia cuando se preguntó por los procesos de traslación en la construcción; “ $\overline{B X}$ es igual a $\overline{A^{\prime} B}$ " (línea 16 del diálogo) fue una observación de la profesora Vicky cuando se preguntó por el segmento $\overline{A^{\prime} B}$.

Adicionalmente, sobre la experimentación, la profesora Elizabeth precisó que

[...] el ejercicio práctico que favorece el doblado de papel y la verificación tangible de la teoría es una bondad que ofrece este colectivo.

(Profesora Elizabeth, 2018).

Veamos las Figuras 11, 12 y 13.

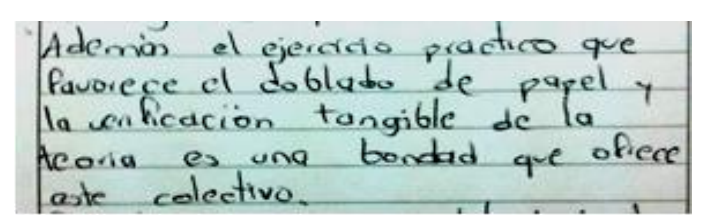

Figura 11 - Material profesora Elizabeth Fuente: construcción personal.

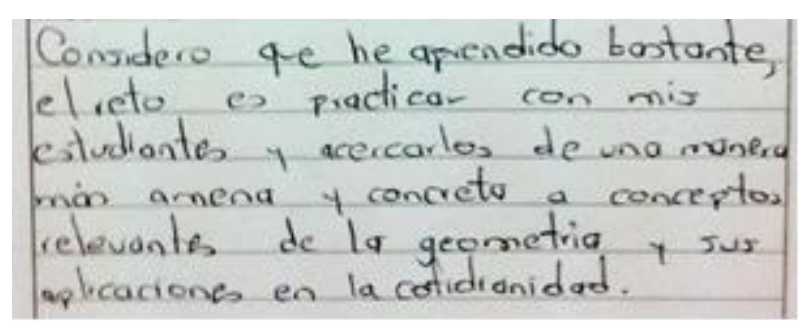

Figura 13 - Material de la profesora Elizabeth Fuente: construcción personal.

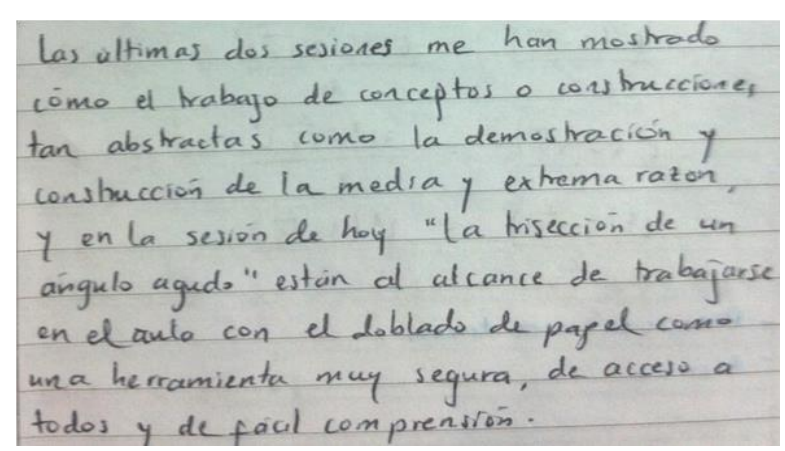

Figura 12 - Material de la profesora Natalia Fuente: construcción personal.

Sin embargo, no solo se puede mencionar que el doblado de papel permite el desarrollo de conocimientos disciplinares de la geometría, sino que también permite el análisis de su conveniencia en el aula de clase (geometría escolar). Al respecto, la profesora Elizabeth mencionó (Figura 13):

[...] considero que he aprendido bastante, el reto es practicar con mis estudiantes y acercarlos de una manera más amena y concreta a conceptos relevantes de la geometría y sus aplicaciones en la cotidianidad.

(Profesora Elizabeth, 2016).

De acuerdo con lo anterior, el uso del doblado de papel en un colectivo de profesores propicia un ambiente que permite la experimentación, la visualización, la generación y validación de conjeturas visuales, la comprensión de conceptos geométricos y el análisis de su 
pertinencia en el aula de clase. Por lo tanto, se convierte en un medio que posibilita la producción de conocimiento geométrico escolar en un colectivo, en tanto que permite la interacción de los profesores para generar una reorganización de su pensamiento (BORBA; VILLARREAL, 2005). Es decir, la cognición humana es de naturaleza social y está condicionada por el uso de diferentes medios o artefactos (VILLARREAL, 2012).

Indicio de categoría 2: interacciones del colectivo de profesores-con-doblado-de-papel en la producción de conocimiento.

La construcción social del conocimiento en colectivos de profesores, fue un tema que emergió durante el desarrollo de la sesión; es decir, se ha encontrado que las profesoras relacionan su formación continua con los aportes recibidos del colectivo. Al respecto, la profesora Elizabeth escribió (Figura 14):

[...] la socialización con mis compañeras me ha generado confianza y ha favorecido una apropiación diferente de los conceptos, teoremas de la geometría euclidiana. Es muy enriquecedor escuchar y debatir sobre alternativas posibles para llegar a la solución de los interrogantes planteados.

(Profesora Elizabeth, 2016).

También se puede percibir, en el fragmento anterior, que su formación parece estar mediada por la confianza que le brindan sus compañeras, lo que le ha generado la apropiación de conocimientos disciplinares sobre la geometría.

De la misma manera, la profesora Natalia, al referirse al aporte del colectivo en cuanto a sus conocimientos disciplinares, agregó que (Figura 15):

[...] refrescar los conocimientos matemáticos y aprenderlos es una de las mayores fortalezas que el colectivo me ha brindado con el doblado de papel como motivo o excusa para este aprendizaje.

(Profesora Natalia, 2016).

Lo anterior, nos permite inferir la tesis de Borba y Villarreal (2005, p. 23) en la que afirman que el conocimiento matemático emerge de un proceso de construcción hecho por un grupo de personas, al que se le llama colectivo, cuando usan determinados medios. Es decir, "el conocimiento es producido por un colectivo compuesto de humanos-con-medios o de humanoscon-tecnologías". En este caso, la producción de conocimiento geométrico escolar emergió de ese colectivo de profesoras, dado que el doblado de papel, como medio, condicionó la naturaleza del conocimiento que se produjo.

Por lo tanto, las profesoras pudieron construir figuras, experimentar, visualizar, expresar conjeturas visuales, dialogar con otras profesoras, probar, aprender conceptos y procedimientos, superar dificultades. Estas interacciones, que se generan al interior del colectivo de profesores-con-doblado-de-papel, permiten la consolidación de ideas relacionadas 
con la geometría escolar y la generación de un ambiente de respeto, de apoyo mutuo y de confianza; estos aspectos se asocian con la producción de conocimiento geométrico escolar, dado que emerge de un colectivo de seres-humanos-con-medios (BORBA; VILLARREAL, 2005).

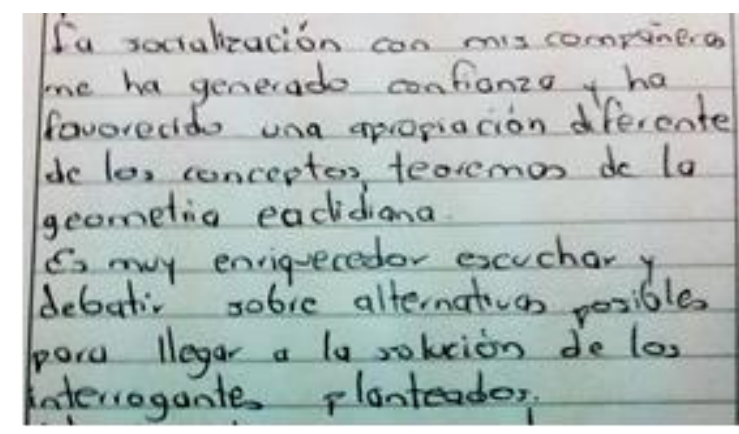

Figura 14 - Material de la profesora Elizabeth Fuente: construcción personal.

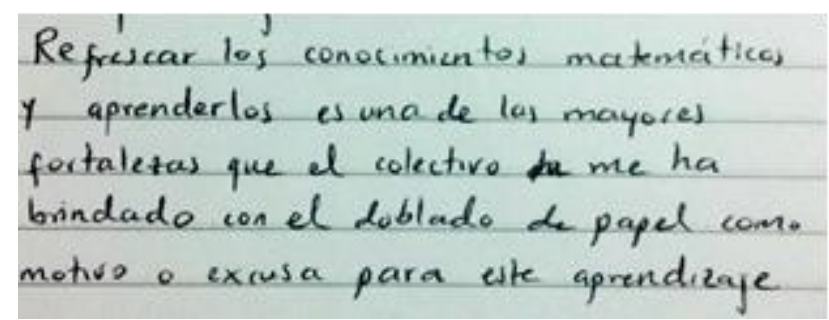

Figura 15 - Material de la profesora Natalia Fuente: construcción personal.

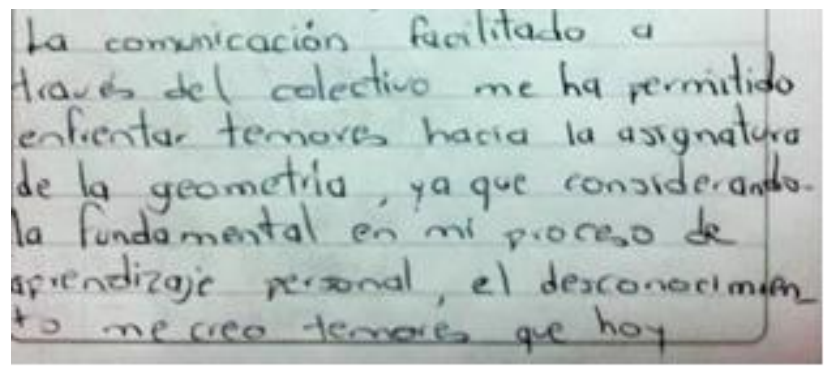

Figura 16 - Material de la profesora Elizabeth Fuente: construcción personal.

\section{Indicio de categoría 3: reflexiones en torno a la práctica pedagógica.}

En las dos categorías anteriores se hizo hincapié en que el uso del doblado de papel y las interacciones del colectivo pueden posibilitar no solo la construcción de ciertos aspectos del conocimiento disciplinar de las matemáticas, particularmente de la geometría en este estudio, sino que también permean el conocimiento de la enseñanza y del aprendizaje de los estudiantes, y la reflexión sobre la práctica pedagógica (geometría escolar). Con relación a este último aspecto, la profesora Elizabeth mencionó que ella había aprendido mucho de la experiencia; de hecho, manifestó que su reto era llevar a la práctica esos conocimientos, para lograr que los estudiantes se acercaran, de manera divertida y concreta, a conceptos geométricos y a sus aplicaciones (Ver figura 13).

Basados en las observaciones que se han registrado, hemos percibido que las tareas de formación que se han trabajado con las profesoras, les ha permitido recordar, apropiarse o aprender conceptos geométricos y, a su vez, intentar analizar su pertinencia en el aula de clase. 
Además, hemos notado motivación para diseñar y aplicar actividades que puedan favorecer el aprendizaje de sus estudiantes. En este sentido, la profesora Natalia mencionó (Figura 17):

[...] cada vez que avanzamos de una actividad a otra los niveles de complejidad van aumentando y por esto aprendemos en cada sesión un reto nuevo que a mi desde lo personal me da motivación de pensar en actividades para llevar al aula de clase.

(Profesora Natalia, 2016).

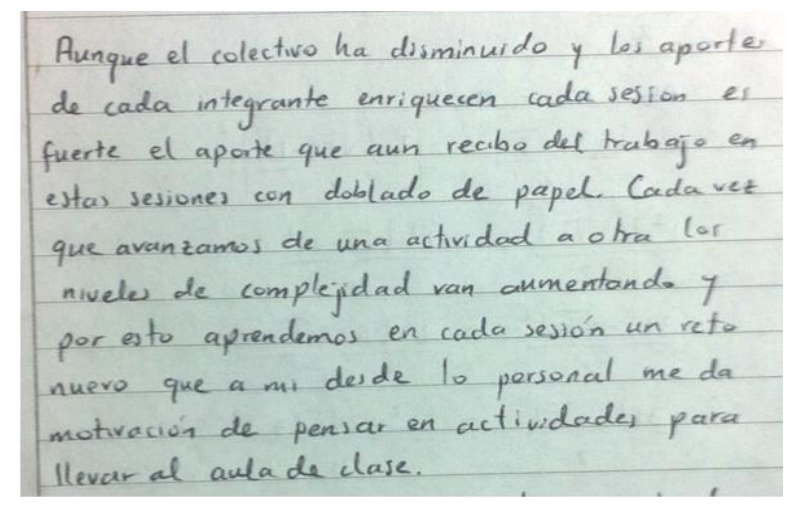

Figura 17 - Material de la profesora Natalia Fuente: construcción personal.

De acuerdo con Ball y Cohen (1999), las tareas de formación deben recrear situaciones auténticas de las prácticas pedagógicas, que les permitan a los profesores acceder, utilizar y desarrollar el conocimiento disciplinar, el de la pedagogía y el de aprendizaje de los estudiantes, de forma simultánea.

Consideramos fundamental resaltar que, en el caso de la profesora Vicky, que no es del área de matemáticas, se percibió un interés y una necesidad marcada de pertenecer al colectivo de profesores-con-doblado-de-papel, dado que deseaba desarrollar potencialidades y nuevos saberes en geometría, a través de las interacciones del mismo colectivo. Ella, de manera informal, explicó a los investigadores y a las demás profesoras, que requería de varios conceptos geométricos para comprender ciertos aspectos de su área y que, por tal razón, se sentía motivada por participar activamente del colectivo.

En este análisis se resalta que la reflexión en torno a la práctica, al rol de ser maestro, se genera dentro del colectivo de profesores-con-doblado-de-papel; este se convirtió en un espacio de aprendizaje, donde cada integrante aportaba desde sus conocimientos, experiencias, necesidades, saberes cotidianos, entre otros. Este espacio fue de confianza, respeto, apoyo mutuo, espontaneidad (FIORENTINI, 2008), reconocimiento del otro, trabajo colaborativo, superación de miedos y colegialidad. Estos aspectos, de índole personal, no son considerados en el constructo teórico de seres-humanos-con-medios de Borba y Villarreal (2005), en tanto que, en este, el sujeto epistémico es un colectivo de humanos-con-medios conformado por humanos, cuya cognición es de naturaleza social, y por medios, artefactos o herramientas, que 
condicionan la producción de conocimiento, y son una componente esencial de dicho sujeto (VILLARREAL, 2012).

\section{Conclusiones}

Con el ánimo de intentar caracterizar la producción de conocimiento geométrico escolar en un colectivo de profesores-con-doblado-de-papel, se desarrolló una tarea de formación con algunas profesoras de una Institución Educativa de la ciudad de Medellín, relacionada con la división de un segmento en media y extrema razón mediante el doblado de papel. Esta tarea está en el marco de la geometría escolar, en tanto que permite que las profesoras discutan conceptos y procedimientos geométricos, mediante el doblado de papel, desde una mirada educativa, es decir, desde sus posturas como docentes.

El análisis de la información que se realizó en el cuarto apartado, permite inferir que la producción de conocimiento geométrico escolar está relacionada con el uso del doblado de papel como medio para visualizar, experimentar y comprender conceptos. De acuerdo con los resultados iniciales de esta investigación, el medio se entiende, de manera más general y más global, como aquel recurso o artefacto (material o inmaterial) que permite la producción de conocimiento y, en general, la reorganización del pensamiento, la cual se puede relacionar con la creación o transformación de esquemas mentales, que pueden ser construidos en colectivos. En consecuencia, tanto el doblado de papel, como el lenguaje verbal o no verbal, fueron tomados como medios que hacen parte activa y constitutiva de la producción de conocimiento geométrico escolar del colectivo de profesores.

También se observó que la producción de conocimiento geométrico escolar parece estar articulada con las interacciones del colectivo de profesores-con-doblado-de-papel. Este colectivo se puede entender como un encuentro entre humanos y medios, que se presenta de manera indisoluble, dado que los medios constituyen al ser humano. Este encuentro permite interacciones entre los humanos con los humanos (a través del lenguaje, que también es un medio), y entre los humanos con los medios (que pueden ser materiales); permite, a través de diálogos, discusiones, procesos de experimentación, procesos de visualización, comprensión de conceptos y procedimientos, producir conocimiento geométrico escolar. Por otro lado, el ambiente de confianza, apoyo mutuo y respeto, que se generó al interior del colectivo, les posibilitó a las profesoras no solo apropiarse de conceptos geométricos, sino también generar ciertos procesos reflexivos sobre su rol como maestras e, incluso, solucionar inquietudes o superar algunos temores con respecto a la enseñanza de la geometría (Ver figura 16). 
El análisis desarrollado también permitió concluir que la producción de conocimiento geométrico escolar se relaciona con la reflexión en torno a la práctica pedagógica. La tarea de formación planteada cumplió con el propósito de profundizar en algunos conocimientos académicos para articularlos con la geometría escolar; así mismo, permitió ciertas reflexiones sobre el uso del doblado de papel en el aula de clase. Cabe resaltar que las interacciones de este colectivo son las que propician el surgimiento de ideas asociadas a la práctica; esto nos permite inferir que las tres categorías, aunque diferenciadas, convergen en la noción de colectivo de profesores-con-doblado-de-papel.

Finalmente, el desarrollo y puesta en marcha del trabajo de campo con las profesoras de la Institución Educativa, nos ha permitido a nosotros, como investigadores, vivenciar discusiones, participar en diálogos, escuchar y compartir experiencias, necesidades, dudas e intereses. Nos ha permitido observar, de primera mano, la motivación de estas por participar, de manera activa, de las situaciones planteadas por el estudio, aun cuando el grupo esté formado por personas de diferentes áreas y experiencias vividas variadas.

De hecho, esas manifestaciones pueden apreciarse en sus producciones escritas dispuestas en sus bitácoras y algunos apartados de los videos. Por lo tanto, formar parte del colectivo de profesores-con-doblado-de-papel, nos ha dado la oportunidad de usar el doblado de papel como medio para visualizar, experimentar y comprender conceptos; nos ha permitido interaccionar con otros profesores o investigadores, para potencializar aprendizajes y, finalmente, nos ha abierto caminos para reflexionar sobre la práctica pedagógica; estos tres aspectos confluyen en el colectivo de profesores-con-doblado-de-papel, que produce algunos conocimientos geométricos escolares.

\section{Referencias}

BALL, D.; COHEN, D. Developing practice, developing practitioners: Toward a practice-based theory of professional education. In: SYKES, G.; DARLING-HAMMOND, L. (Ed.). Teaching as the learning profession: Handbook of policy and practice. San Francisco: Jossey Bass, 1999. p. 3-32.

BORBA, M. Tecnologias Informáticas na Educação Matemática e Reorganização de Pensamento. In: BICUDO, M. (Org.). Pesquisa em Educação Matemática: Concepções \& Perspectivas. São Paulo: Editora UNESP, 1999. p. 285-295.

BORBA, M. Humans-with-media and continuing education for mathematics teachers in online environments. ZDM Mathematics Education, Berlín, v. 44, n. 6, p. 801-814, 2012

BORBA, M.; SCUCUGLIA, R.; GADANIDIS, G. Fases das tecnologias digitais em educação matemática. Sala de aula e internet em movimento. Belo Horizonte: Autêntica, 2014. 
BORBA, M.; VILLARREAL, M. Humans-with-Media and the reorganization of Mathematical Thinking. New York: Springer, 2005.

FIORENTINI, D. ¿Investigar prácticas colaborativas o investigar colaborativamente? In: BORBA, M.; ARAÚJO, J. (Ed.). Investigación Cualitativa en Educación Matemática. Balderas: Limusa, 2008. p. 43-72.

HERNÁNDEZ, R.; FERNÁNDEZ, C.; BAPTISTA, P. Metodología de la Investigación. México D. F.: McGraw Hill, 2006.

LÉVY, P. As tecnologias da inteligencia. O futuro do pensamento nu era da Informática. Traducción COSTA, C. Les technologies de I'intelligence. San Pablo: Editora 34, 1993.

MOLINA, M. et al. Un acercamiento a la investigación de diseño a través de los experimentos de enseñanza. Enseñanza de las Ciencias, Barcelona, v. 29, n. 1, p. 75-88, 2011.

MONSALVE, O; JARAMILLO, C. El placer de doblar papel. Mostraciones y algunas aplicaciones matemáticas. Revista Educación y Pedagogía, Medellín, v. XV, n. 35, p. 11-25, 2003.

MOREIRA, P.; DAVID, M. A formação matemática do professor. Licenciatura e prática docente escolar. Belo Horizonte: Autêntica, 2005.

PONTE, J. et al. Tools and Settings Supporting Mathematics Teachers' Learning in and from Practice. In: EVEN, R.; BALL, D. (Ed.). The Professional Education and Development of Teachers of Mathematics. New York: Springer, 2009. p. 185-209.

RODRÍGUEZ, G.; GIL, J.; GARCÍA, E. Metodología de la investigación cualitativa. Málaga: Algibe, 1999.

ROW, S. Geometric Exercises in Paper Folding. New York: Dover Publications, 1966.

SANTA, Z.; JARAMILLO, C. Aplicaciones de la geometría del doblado de papel a las secciones cónicas. Revista Virtual Universidad Católica del Norte, Medellín, n. 31, p. 338-362. 2010.

Disponible en: <http://revistavirtual.ucn.edu.co/index.php/RevistaUCN/article/view/48/105>. Acceso en: 05 jun. 2017.

SANTA, Z.; JARAMILLO, C. Producción de conocimiento geométrico de un colectivo-con-doblado de papel: el caso de la trisección de un ángulo. In: COMITÉ INTERAMERICANO DE EDUCACIÓN MATEMÁTICA, 14., 2015, Chiapas. Memorias de la XIV CIAEM. Chiapas: Comité Interamericano de Educación Matemática, 2015. p. 1-12.

SANTA, Z.; JARAMILLO, C.; BORBA, M. Processos de produção de conhecimento geométrico na construção da média e extrema razão. In: SOCIEDADE BRASILEIRA DE EDUCAÇÃ̃O MATEMÁTICA, 4., 2015, Ilhéus. Anais do $4^{\circ}$ Simpósio Internacional de Pesquisa em Educação Matemática. Ilhéus: Universidade Estadual de Santa Cruz UESC, 2015. p. 3161-3166.

SOUTO, D. Transformações expansivas em um curso de educação matemática a distância online. 2013. 281f. Tese (Doutorado em Educação Matemática) - Instituto de Geociências e Ciências Exatas, Universidade Estadual Paulista "Júlio de Mesquita Filho", Rio Claro, 2013.

TIKHOMIROV, O. The psychological consequences of computerization. In: Wertsch, J. (Ed.). The concept of activity in Soviet Psychology. New York: M. E. Sharpe Inc, 1981. p. 256-278. 
VILLA-OCHOA, J.; RUIZ, M. Pensamiento variacional: seres-humanos-con-GeoGebra en la visualización de nociones variacionales. Revista Educ. Matem. Pesq., São Paulo, v. 12, n. 3, p. 514$528,2010$.

VILLARREAL, M.; BORBA, M. Collectives of humans-with-media in mathematics education: notebooks, blackboards, calculators, computers and... notebooks throughout 100 years of ICMI. ZDM Mathematics Education, Berlín, v. 42, n. 1, p. 49-62, 2010.

VILLARREAL, M. Tecnologías y educación matemática: necesidad de nuevos abordajes para la enseñanza. In: Virtualidad, Educación y Ciencia VEsC, Córdoba, v. 3, n. 5, p. 73-94, 2012.

Submetido em 07 de Julho de 2017. Aprovado em 30 de Abril de 2018. 